\title{
CORRECTION
}

\section{Correction to: Respect for the journey: a survivor-led investigation of undergoing psychotherapy assessment}

\author{
Alison Faulkner ${ }^{1} \cdot$ Katie Kelly $^{2} \cdot$ Sarah Gibson $^{3} \cdot$ Steve Gillard $^{4} \cdot$ Lana Samuels $^{5} \cdot$ Angela Sweeney $^{6} \oplus$
}

(C) The Author(s) 2021

\section{Correction to: Social Psychiatry and Psychiatric Epidemiology https://doi.org/10.1007/s00127-020-02017-1}

The original version of this article contained references to articles and authors that were blinded. These should read as follows:

Semi-structured interview schedules were developed through reflecting on our experiential knowledge, informed by narrative (Sweeney et al. 2016) and systematic reviews (Sweeney et al. 2019).

SUAG members contributed to all aspects of the study, with the detailed study design guided by a service user Ethics Working Group (authors 1, 2, 3 \& 5).
Interviews were conducted in 2018 by a survivor researcher (author 6).

The original article has been corrected.
The original article can be found online at https://doi.org/10.1007/ s00127-020-02017-1.

\section{Angela Sweeney}

angela.sweeney@kcl.ac.uk

Alison Faulkner

alison.faulkner2@btinternet.com

Katie Kelly

Katie.bogart@nhs.net

Sarah Gibson

gibsons2go@gmail.com

Steve Gillard

Steven.Gillard@city.ac.uk

Lana Samuels

1samuels_9224it@yahoo.co.uk
Independent Researcher, London, UK

2 Tower Hamlets Early Intervention for Psychosis Service (THEIS), East London NHS Foundation Trust, London E2 6BF, UK

3 Independent Researcher, Kent, England

4 School of Health Sciences, City, University of London, London EC1V 0HB, UK

5 PEER, St George's, University of London, Cranmer Terrace, London SW17 0RE, UK

6 Service User Research Enterprise, King's College London, London SE5 8AF, UK 